\title{
Half-Magnetic Topological Insulator with Magnetization-Induced Dirac Gap at a Selected Surface
}

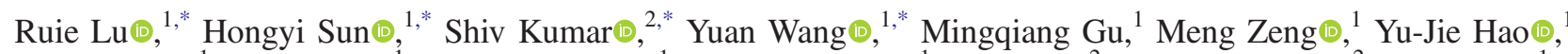 \\ Jiayu Li®, ${ }^{1}$ Jifeng Shao, ${ }^{1}$ Xiao-Ming Ma $\odot,{ }^{1}$ Zhanyang Hao $\odot,{ }^{1}$ Ke Zhang, ${ }^{2}$ Wumiti Mansuer, ${ }^{2}$ Jiawei Mei, ${ }^{1}$

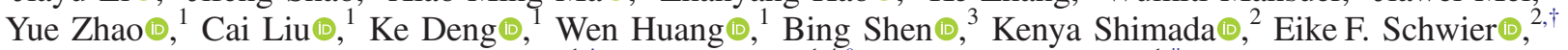 \\ Chang Liu $\odot,{ }^{1, \$}$ Qihang Liu $\odot,{ }^{1,4, \S}$ and Chaoyu Chen $\oplus^{1, \|}$ \\ ${ }^{1}$ Shenzhen Institute for Quantum Science and Engineering (SIQSE) and Department of Physics, \\ Southern University of Science and Technology (SUSTech), Shenzhen 518055, China \\ ${ }^{2}$ Hiroshima Synchrotron Radiation Centre, Hiroshima University, \\ Higashi-Hiroshima, Hiroshima 739-0046, Japan \\ ${ }^{3}$ School of Physics, Sun Yat-Sen University, Guangzhou 510275, China \\ ${ }^{4}$ Guangdong Provincial Key Laboratory for Computational Science and Material Design, \\ Southern University of Science and Technology, Shenzhen 518055, China
}

(Received 9 September 2020; revised 17 November 2020; accepted 13 January 2021; published 25 February 2021)

\begin{abstract}
Topological magnets are a new family of quantum materials providing great potential to realize emergent phenomena, such as the quantum anomalous Hall effect and the axion-insulator state. Here, we present our discovery that the stoichiometric ferromagnet $\mathrm{MnBi}_{8} \mathrm{Te}_{13}$ with natural heterostructure $\mathrm{MnBi}_{2} \mathrm{Te}_{4} /\left(\mathrm{Bi}_{2} \mathrm{Te}_{3}\right)_{3}$ is an unprecedented "half-magnetic topological insulator," with the magnetization existing at the $\mathrm{MnBi}_{2} \mathrm{Te}_{4}$ surface but not at the opposite surface terminated by triple $\mathrm{Bi}_{2} \mathrm{Te}_{3}$ layers. Our angle-resolved photoemission spectroscopy measurements unveil a massive Dirac gap at the $\mathrm{MnBi}_{2} \mathrm{Te}_{4}$ surface and a gapless Dirac cone on the other side. Remarkably, the Dirac gap (about $28 \mathrm{meV}$ ) at the $\mathrm{MnBi}_{2} \mathrm{Te}_{4}$ surface decreases monotonically with increasing temperature and closes right at the Curie temperature, thereby representing the first smoking-gun spectroscopic evidence of a magnetizationinduced topological surface gap among all known magnetic topological materials. We further demonstrate theoretically that the half-magnetic topological insulator is desirable to realize the surface anomalous Hall effect, which serves as direct proof of the general concept of axion electrodynamics in condensed matter systems.
\end{abstract}

DOI: $10.1103 /$ PhysRevX.11.011039

Subject Areas: Condensed Matter Physics

\section{INTRODUCTION}

Magnetic topological insulators (TI) showcase quantum magnetism and nontrivial band topology, thereby providing a unique playground for exploring exotic quantum phenomena in condensed matter physics [1-16]. One

\footnotetext{
* These authors contributed equally to this work.

Corresponding author.

Eike.schwier@physik.uni-wuerzburg.de

Corresponding author.

liuc@sustech.edu.cn

${ }^{\S}$ Corresponding author.

liuqh@sustech.edu.cn

"Corresponding author.

chency@ sustech.edu.cn

Published by the American Physical Society under the terms of the Creative Commons Attribution 4.0 International license. Further distribution of this work must maintain attribution to the author(s) and the published article's title, journal citation, and DOI
}

paradigmatic example is the so-called axion-insulator phase, which exhibits bulk topological magnetoelectric response with the phase angle $\theta=\pi$ protected by either inversion or time-reversal symmetry $[10,15,16]$. The resultant bulk-boundary correspondence is the predicted halfquantized surface Hall conductance $e^{2} / 2 h$ in the absence of an external magnetic field given that a magnetic gap exists at the surface. The intrinsic magnetic TI $\mathrm{MnBi}_{2} \mathrm{Te}_{4} /\left(\mathrm{Bi}_{2} \mathrm{Te}_{3}\right)_{n}(n=1,2,3, \ldots)$, comprising alternating layers of magnetic $\mathrm{TI} \mathrm{MnBi}_{2} \mathrm{Te}_{4}$ and nonmagnetic $\mathrm{TI} \mathrm{Bi}_{2} \mathrm{Te}_{3}$, has the potential for realizing both the quantum anomalous Hall $(\mathrm{QAH})$ insulator and the axion-insulator phases [1-4,17-22]. In principle, these compounds are ideal candidates for the inversion-preserved axion insulators with a persistent surface gap originating from the long-range magnetic order. However, it remains controversial whether the topological surface states (TSS) of $\mathrm{MnBi}_{2} \mathrm{Te}_{4} /$ $\left(\mathrm{Bi}_{2} \mathrm{Te}_{3}\right)_{n}$ are gapped or gapless [23-31]. Recent angleresolved photoemission spectroscopy (ARPES) measurements unexpectedly revealed an almost gapless surface Dirac cone in $\mathrm{MnBi}_{2} \mathrm{Te}_{4}$ and $\mathrm{MnBi}_{2} \mathrm{Te}_{4}$ termination of 
(a)

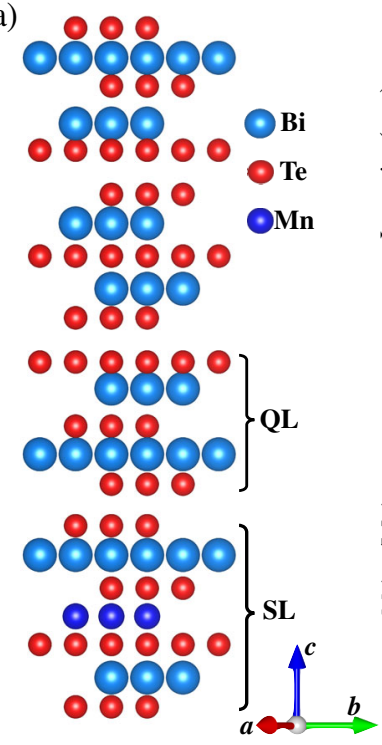

(b)

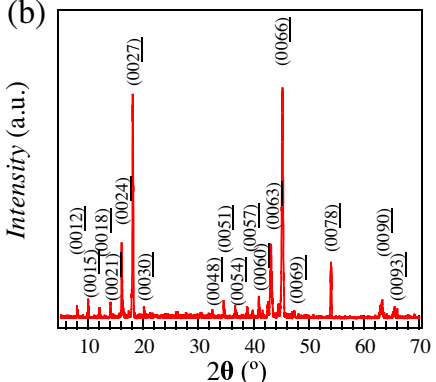

(e)

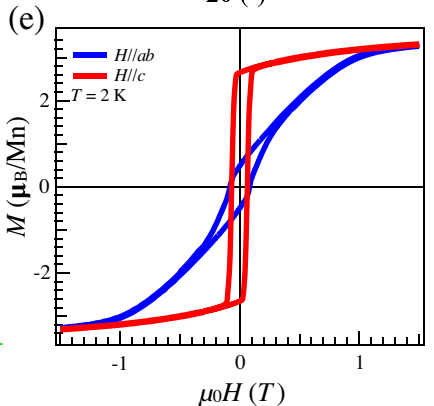

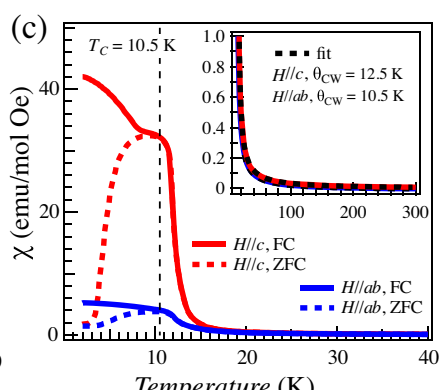
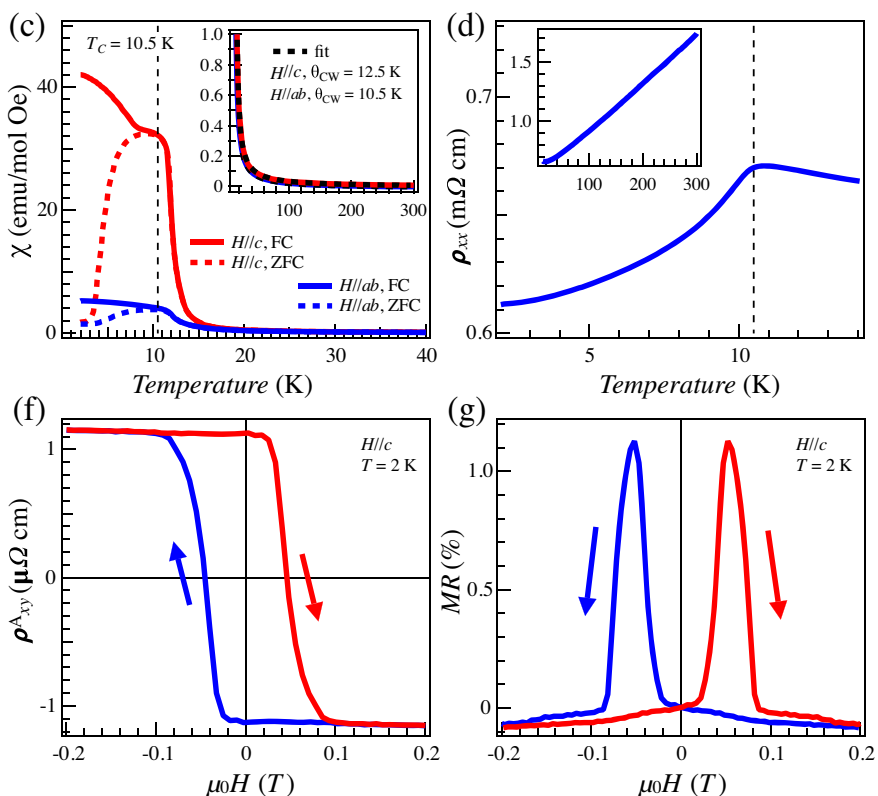

FIG. 1. Ferromagnetism and anomalous Hall effect in $\mathrm{MnBi}_{8} \mathrm{Te}_{13}$ single crystals. (a) Schematic crystal structure with one unit of -SL-QL-QL-QL- sequences. (b) Single-crystal x-ray diffraction result taken at $300 \mathrm{~K}$. (c) Zero-field-cooled (ZFC, dashed line) and field-cooled (FC, solid line) magnetic susceptibility $(\chi)$ vs temperature $(T)$ for magnetic field $H=100$ Oe parallel to the $a b$ plane (blue) and the $c$ axis (red), respectively. The inset shows the Curie-Weiss fitting for a temperature range of $150 \mathrm{~K}-300 \mathrm{~K}$. (d) Zero-field inplane resistivity $\left(\rho_{\mathrm{xx}}\right)$ vs $T$. The inset shows the results up to $300 \mathrm{~K}$. (e) Field-dependent magnetization hysteresis at $2 K$ for $H / / a b$ (blue) and $H / / c$ (red). (f) Field-dependent anomalous Hall resistivity $\left(\rho_{x y}^{A}\right)$ at $2 K$ for $H / / c$. (g) Field-dependent transverse resistivity change $\left(M R=\left[\rho_{x x}(H) / \rho_{x x}(0)\right]-1\right)$ at $2 K$ for $H / / c$.

$\mathrm{MnBi}_{4} \mathrm{Te}_{7}$ [32-34], which may be attributed to reduced effective magnetic moments due to the extension of the surface states to the bulk $[28,35]$. On the other hand, various surface gaps have been reported in this material family $[1,2,25,35]$, where the speculated mechanisms of the gap opening are from magnetization, hybridization, dephasing, Coulomb scattering, and so on. Furthermore, none of these reported gaps has been directly proved to originate from magnetic orders since they remain open within the hightemperature paramagnetic (PM) phase [25,35-37], which makes the realization of the axion-insulator phase obsolete.

Here, we conduct ARPES measurements on stoichiometric $\mathrm{MnBi}_{8} \mathrm{Te}_{13}\left[\mathrm{MnBi}_{2} \mathrm{Te}_{4} /\left(\mathrm{Bi}_{2} \mathrm{Te}_{3}\right)_{n}\right.$ with $\left.n=3\right]$, with an intrinsic ferromagnetic (FM) ground state. At the $\mathrm{MnBi}_{2} \mathrm{Te}_{4}$ septuple-layer (SL) termination, we have directly revealed a surface gap of about $28 \mathrm{meV}$ below the Curie temperature of $T_{C}=10.5 \mathrm{~K}$, which decreases monotonically with increasing temperature and closes right at $T_{C}$ to form a gapless Dirac cone, proving its magnetic nature. These results represent the first direct spectroscopic evidence of a magnetization-induced topological surface gap among all known magnetic topological materials. In sharp contrast, a gapless Dirac cone with negligible FM proximity is observed on the opposite surface terminated by the triple $\mathrm{Bi}_{2} \mathrm{Te}_{3}$ quintuple layers (QL), analogous to the situation in nonmagnetic $\mathrm{TI}_{\mathrm{Bi}_{2}} \mathrm{Te}_{3}$. Recalling that a typical magnetic TI manifests magnetic gaps at both surfaces (e.g., top and bottom) perpendicular to the magnetic moment, here we refer to the ferromagnet $\mathrm{MnBi}_{8} \mathrm{Te}_{13}$ as a "half-magnetic TI," in which the $\mathrm{MnBi}_{2} \mathrm{Te}_{4}$ SL surface is gapped by surface magnetization while the opposite triple- $\mathrm{Bi}_{2} \mathrm{Te}_{3} \mathrm{QL}$ surface remains nonmagnetic and thus gapless. Utilizing density functional theory (DFT) calculations, we find halfQAH conductivity well localized at the SL termination, regardless of the cleavage of the other termination. Therefore, the half-magnetic TI provides an ideal platform for observing the half-QAH effect at a single surface and the related axion physics.

\section{II. $\mathrm{MnBi}_{8} \mathrm{Te}_{13}$ SINGLE CRYSTAL WITH FM GROUND STATE}

Single-crystal $\mathrm{MnBi}_{8} \mathrm{Te}_{13}$ has a trigonal structure [38], with a space group of $R \overline{3} \mathrm{~m}$. The lattice of $\mathrm{MnBi}_{8} \mathrm{Te}_{13}$ consists of one $\mathrm{MnBi}_{2} \mathrm{Te}_{4}$ SL and three $\mathrm{Bi}_{2} \mathrm{Te}_{3}$ QLs stacking alternately along the $c$ axis [Fig. 1(a)]. These SLs or QLs are coupled through weak van der Waals $(\mathrm{vdW})$ forces. Cleaving the single crystal perpendicular to the $c$ axis could yield four possible terminations, namely, the $\mathrm{S}$ termination, Q termination, QQ termination, and QQQ termination. The crystallinity was examined by X-ray diffraction (XRD). As shown in Fig. 1(b), all of the diffraction peaks, particularly the low-angle ones, can be well indexed by the $(00 l)$ reflections with lattice parameter $c \approx 132.6 \AA$ (calculated from the Rietveld 
refinement of powder XRD, as shown in Fig. S1 in the Supplemental Material [39]), in agreement with previous work [40].

The temperature-dependent anisotropic magnetic susceptibility [Fig. 1(c)] shows Curie-Weiss (CW) behavior above $150 \mathrm{~K}$ (inset) with the characteristic temperature $\theta_{C W}=12.5 \mathrm{~K}$ and $10.5 \mathrm{~K}$ for $H / / c$ and $H / / a b$, respectively, through a fitting with $\chi(T)=\chi_{0}+C /\left(T-\theta_{\mathrm{CW}}\right)$. Around $T_{C}=10.5 \mathrm{~K}$, a FM transition was revealed by magnetic susceptibility [Fig. 1(c)] and resistivity measurements [Fig. 1(d)]. The frustration parameter $\left(\theta_{C W} / T_{C}\right)$ for $H / / c$ was calculated to be about 2 , indicating a moderate magnetic frustration. For $H / / c$, the observed larger bifurcation between zero-field cooling (ZFC) and field cooling (FC) magnetization [Fig. 1(c)] and magnetic hysteresis loop [Fig. 1(e)] indicate an easy axis along the $c$ axis and an Ising-type exchange interaction between adjacent $\mathrm{Mn}$ layers. The saturation moment $M_{s a}=3.58 \mu_{B} / \mathrm{Mn}$ is close to that of $3.56 \mu_{B} / \mathrm{Mn}$ in $\mathrm{MnBi}_{2} \mathrm{Te}_{4}$ [41] and 3.5 $\mu_{B} / \mathrm{Mn}$ in $\mathrm{MnBi}_{4} \mathrm{Te}_{7}$ [29]. The above magnetic properties suggest a FM order with an out-of-plane magnetic moment configuration in $\mathrm{MnBi}_{8} \mathrm{Te}_{13}$, in contrast to the A-type $\mathrm{AFM}$ ground states found in other $\mathrm{MnBi}_{2} \mathrm{Te}_{4} /\left(\mathrm{Bi}_{2} \mathrm{Te}_{3}\right)_{n}$ compounds $(n=0,1,2)[42,43]$.

The field-dependent Hall resistivity $\left[\rho_{x y}(H)\right]$ and magnetoresistivity $\left[\mathrm{MR}=\left[\rho_{x x}(H) / \rho_{x x}(0)\right]-1\right]$ are shown in Figs. 1(f) and 1(g), as well as Fig. S3 in the Supplemental Material [39]. The negative slope of $\rho_{x y}(H)$ in Fig. S3(f) indicates electron-type carriers, and the obvious anomalous Hall effect is observed for $H / / c$. In a ferromagnet, the Hall resistivity is described by the formula $\rho_{x y}=R_{0} H+\rho_{x y}^{A}=R_{0} H+R_{s} M$, where $R_{0}$ is the ordinary Hall coefficient, $\rho_{x y}^{A}$ is the anomalous Hall resistivity, $R_{s}$ is the anomalous Hall coefficient, and $M$ is the magnetization. Above $T_{C}(20 \mathrm{~K}), \rho_{x y}(H)$ exhibits the same slope at all $H$ [see Fig. S3(f)], indicating a constant $R_{0}$, which allows us to subtract the ordinary Hall resistivity to obtain the anomalous part, as shown in Fig. 1(f). Note that $R_{s}$ scales well with the $M-H$ curve to the anomalous part of the Hall resistivity and is calculated to be $R_{s}=1.76 \times 10^{-6} \mathrm{~m}^{3} / \mathrm{C}, 2$ orders of magnitude larger than $R_{0}=1.15 \times 10^{-8} \mathrm{~m}^{3} / \mathrm{C}$. Unlike the previous report [40], the MR from both increasing and decreasing field measurements maintains a near-vanishing value $(<0.1 \%)$ and exhibits sharp peaks without any overlap at the appearance of the anomalous Hall plateau. This feature is reminiscent of the MR behavior in Cr-doped $(\mathrm{Bi}, \mathrm{Sb})_{2} \mathrm{Te}_{3}$ films when approaching the quantum anomalous Hall region $[11,12]$.

\section{GAPPED AND GAPLESS TSS DIRAC CONE IN MnBi $\mathrm{Te}_{13}$}

We employ a $\mu$-laser-ARPES system [44], with a focused laser spot size of about $5 \mu \mathrm{m}$, to measure the termination-sensitive band structure of $\mathrm{MnBi}_{8} \mathrm{Te}_{13}$. Figures S4 and S5 present the spectra in a highsymmetry direction, as well as a set of constant energy contours for all four terminations. In Fig. 2, we highlight the band structure of the $\mathrm{S}$ termination and its opposite cleaving plane, the QQQ termination. Shown are spectra taken at $7 \mathrm{~K}$ and $20 \mathrm{~K}$, which correspond to FM and PM phases, respectively. In the FM phase, the S termination shows an unambiguous energy gap of about $28 \mathrm{meV}$ at the Dirac point [Fig. 2(c)], which is in sharp contrast to other Mn-Bi-Te family members such as $\mathrm{MnBi}_{2} \mathrm{Te}_{4} \quad[30,32-34], \quad \mathrm{MnBi}_{4} \mathrm{Te}_{7} \quad[24,30,36]$, and $\mathrm{MnBi}_{6} \mathrm{Te}_{10}$ [28,30], whose $\mathrm{S}$ terminations consistently show no apparent gap opening at the Dirac point below the magnetic transition [45]. Above $T_{C}$ in the PM phase, a gapless Dirac cone is observed [Fig. 2(d)]. Comparison between the FM and PM phases suggests that the origin of the surface gap for the $S$ termination is magnetism. The gap opening is captured by an effective massive Dirac Hamiltonian $H_{\text {surf }}(k)=\left(\sigma_{x} k_{y}-\sigma_{y} k_{x}\right)+$ $m_{\text {eff }} \sigma_{z}$, where the first two terms describe a Dirac cone and the last the effective Zeeman field induced by the ferromagnetically ordered $\mathrm{Mn}$ atoms. The gap size, $m_{\text {eff }} \sim 28 \mathrm{meV}$, is in qualitative agreement with our DFT prediction [Fig. 2(b)]. The detailed comparison between the DFT and the ARPES results is provided in Fig. S4.

At the QQQ termination, the gapless Dirac surface states appear to persist below $T_{C}$. At first sight, this case seems to contradict the broken time-reversal symmetry. However, given the considerable spatial separation between the top $\mathrm{Bi}_{2} \mathrm{Te}_{3} \quad \mathrm{QL}$ and the magnetic $\mathrm{MnBi}_{2} \mathrm{Te}_{4} \mathrm{SL}$, it is reasonable to assume a negligibly small effective Zeeman field for the surface states. Such a conjecture is indeed supported by our DFT calculation, which also reproduces the gapless Dirac cone despite a magnetic ground state [Fig. 2(f)]. Here, we note that the DFT surface-only spectra agree with the ARPES spectra better than the DFT bulk spectra, likely because of the limited photoemission probing depth [47]. Similar gapless Dirac cones have been observed by ARPES at the FM phase for both Q and QQ terminations, owing to the hybridization between the TSS and the bulk bands that buries the Dirac point [28], shown in Fig. S6. To sum up, $\mathrm{MnBi}_{8} \mathrm{Te}_{13}$ is a half-magnetic topological insulator. The time-reversal symmetry is broken at the $\mathrm{S}$ termination, which shows a temperature-dependent gap, while it is approximately preserved at the other surface, which shows a gapless state.

Furthermore, in contrast to the unambiguous temperature evolution of the TSS, we find the band-structure change of the bulk states between the FM [Fig. 2(c), $7 \mathrm{~K}$ ] and PM [Fig. 2(d), $20 \mathrm{~K}$ ] to be negligible, which is probably attributed to the reduced effective magnetic moments that the particular bulk band feels. 

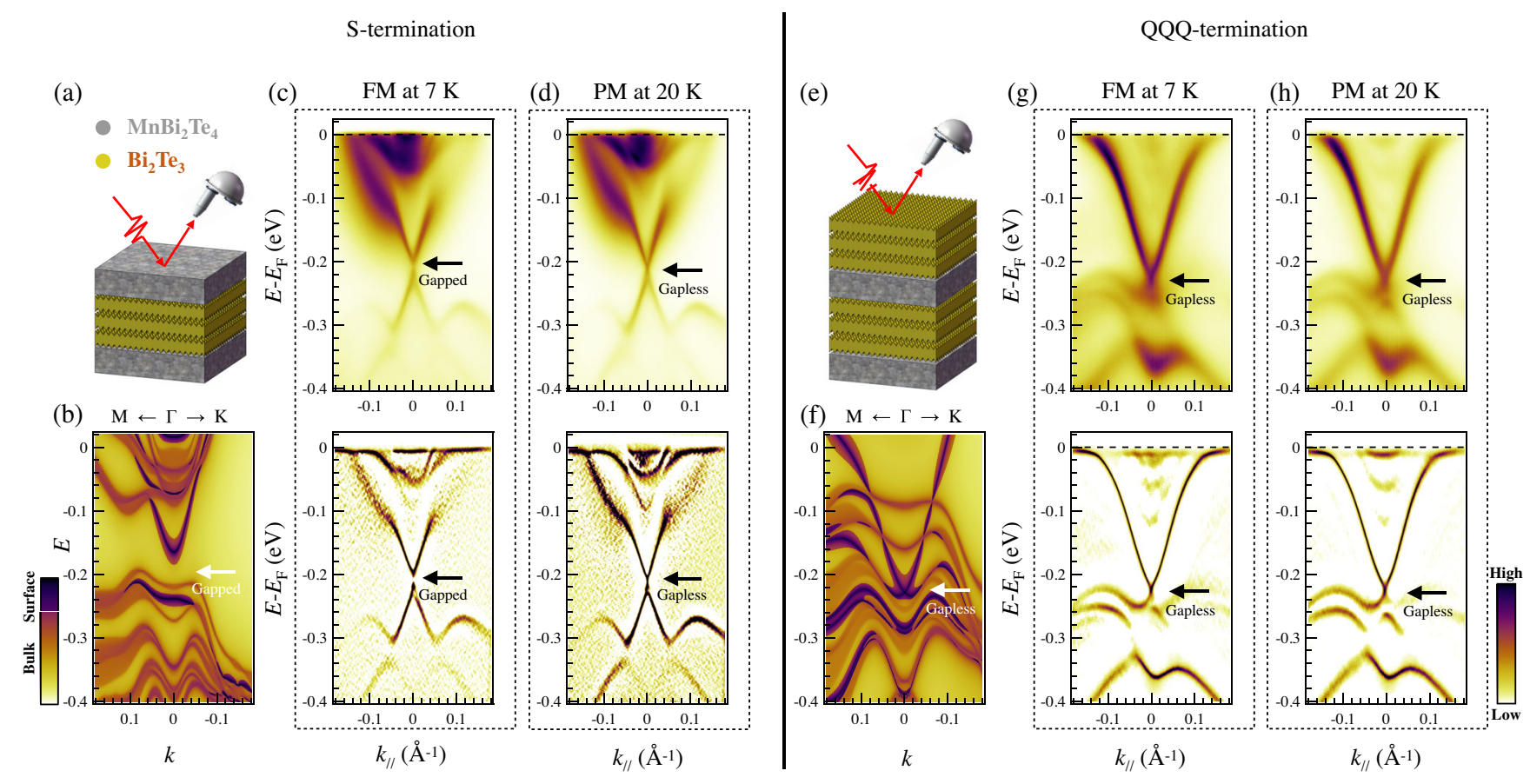

FIG. 2. Temperature evolution of the TSS Dirac cone gap at the $S$ and QQQ termination of $\mathrm{MnBi}_{8} \mathrm{Te}_{13}$. (a)-(d) ARPES results for the $\mathrm{S}$ termination. (a) Schematic structural stacking configuration of the S termination. (b) DFT band structure calculated for the FM state, with the surface-only states (dark violet to black) being superposed on the bulk states (yellow, orange, and light violet). (c) Band structure along $\bar{M}-\bar{\Gamma}-\bar{M}$ measured at $7 \mathrm{~K}$ (FM state). (d) Band structure along $\bar{M}-\bar{\Gamma}-\bar{M}$ measured at $20 \mathrm{~K}$ (PM state). Data in panels (c) and (d) are shown in the form of the ARPES original spectra (top panel) and the 2D curvature spectra [46] (bottom panel). (e)-(h) Same as panels (a)-(d) but for the QQQ termination. Clearly, entering the FM state opens a gap at the TSS Dirac cone for the S termination, while no such gap is observed for the QQQ termination.

\section{NATURE OF THE TSS DIRAC GAP AT S TERMINATION}

Having established a TSS Dirac-point gap opening in the $\mathrm{S}$ termination of $\mathrm{FM} \mathrm{MnBi}_{8} \mathrm{Te}_{13}$, we now demonstrate that this gap is indeed opened because of the long-range FM order of the magnetic moments. A zoomed-in ARPES $k-E$ map of the $\mathrm{S}$ termination in the PM state $(15 \mathrm{~K})$ is shown in Fig. 3(a), while that in the FM state (7K) is shown in Fig. 3(d). The magnetic-order-induced spectral change is concentrated at the Dirac point, as highlighted in Figs. 3(b) and 3(e). For the PM S termination, a gapless "X"-shape Dirac cone can be clearly resolved, with its Dirac point being indicated by the black arrow in Fig. 3(b). The corresponding energy distribution curve (EDC) taken across the Dirac point can be fitted with two Lorentzian peaks, with the dominating one (dark blue) centered at the Dirac-point energy $E_{D} \approx-0.21 \mathrm{eV}$ [Fig. 3(c)].

For the FM S termination, as presented in Fig. 3(e), the upper and lower Dirac cones are separated in energy by a sizable gap, with the two cones clearly detaching from each other. Fitting the corresponding EDC yields three Lorentzian peaks. The two dark-blue peaks, located at $E_{1} \approx$ $-0.19 \mathrm{eV}$ and $E_{2} \approx-0.22 \mathrm{eV}$, correspond to the upper Dirac cone minimum and lower Dirac cone maximum, respectively. These two peaks originate from the splitting of the gapless Dirac-point peak centered at $E_{D} \approx-0.21 \mathrm{eV}$ [Fig. 3(c)], resulting in a Dirac-point gap of $\Delta=E_{1}-E_{2} \approx$ $28 \mathrm{meV}$ [Fig. 3(f)]. The light-blue peak corresponds to a weak shoulder found in both PM and FM EDCs, whose peak position remains at the same energy at different temperatures and potentially originates from disorder.

In Fig. 3(g), systematic Lorentzians fitting to the $\bar{\Gamma}$ EDCs at various temperatures below and above the bulk PM-FM transition $(10.5 \mathrm{~K})$ are presented. The constant energy mapping and dispersions corresponding to each temperature are shown in Figs. S7 and S8, allowing us to unambiguously extract the dispersion at the $\Gamma$ point. At the lowest temperature $(6 \mathrm{~K})$, similarly, three Lorentzian peaks are needed to fit the EDC, of which two dark-blue peaks $\left(E_{1}\right.$ and $\left.E_{2}\right)$ correspond to the split Dirac cone. The Dirac cone gap size $\Delta=E_{1}-E_{2}$, and its temperature evolution is plotted in Fig. 3(h). With increasing temperature, $E_{1}$ and $E_{2}$ move closer to each other ( $\Delta$ decreases) and eventually merge into one Lorentzian peak at $11 \mathrm{~K}$ (gap closes), strongly suggesting a clear correlation between the size of this Dirac-point gap and the FM exchange interaction. It is worth noting that, while, similarly, we can also assume two dark-blue peaks $\left(E_{1}\right.$ and $\left.E_{2}\right)$ for the EDCs measured at $T \geq 11 \mathrm{~K}$, the fitting iterations always result in 

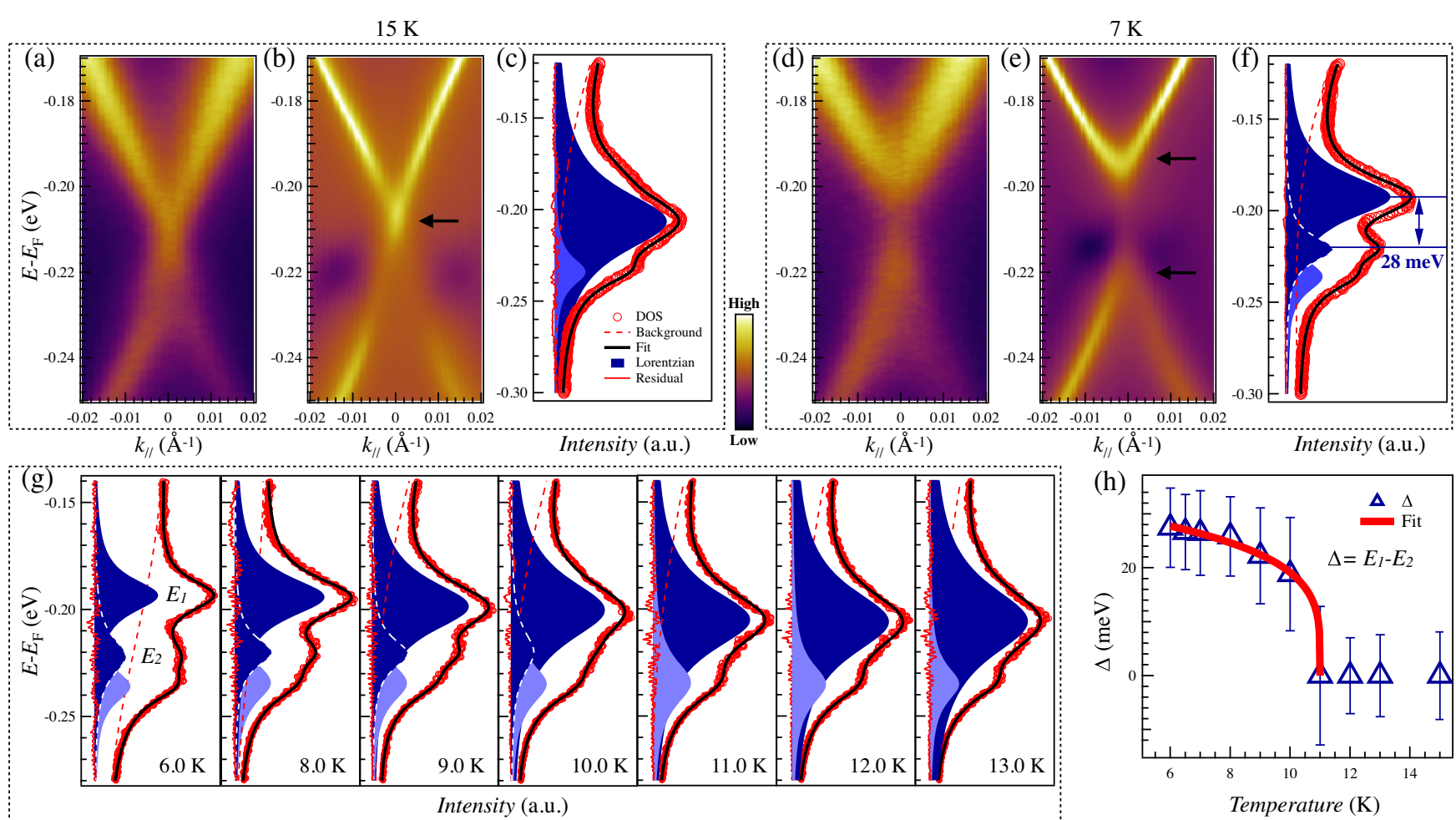

FIG. 3. Temperature dependence of the TSS gap in the $\mathrm{S}$ termination of $\mathrm{MnBi}_{8} \mathrm{Te}_{13}$. (a) Enlarged ARPES spectrum of the $\mathrm{S}$ termination measured at $15 \mathrm{~K}$ (PM phase). (b) Two-dimensional curvature plot for the spectrum in panel (a). (c) Energy distribution curve (EDC) at $\bar{\Gamma}$ (integrated over a $\pm 0.001 \AA^{-1}$ momentum window) and its fitting with multiple Lorentzian peaks. (d)-(f) Same plots as those in panels (a)-(c) but for data measured at $7 \mathrm{~K}$ (FM phase). (g) EDC fitting analysis at various temperatures, showing a clear gap opening for $\mathrm{T} \leq 10.0 \mathrm{~K}$. The EDCs for each temperature are extracted from the corresponding spectra shown in Fig. S6 integrated over a $\pm 0.001 \AA^{-1}$ momentum $k_{\|}$window. Furthermore, the spectra for each temperature are extracted from the corresponding map shown in Fig. S5 integrated over a $\pm 0.002 \AA^{-1}$ momentum $\left(k_{y}\right)$ window. (h) TSS Dirac cone gap size (blue triangles) evolution with temperature and its fitting (red solid line) using a power-law curve. The error bar of the gap size is defined as $e=\sqrt{w_{1}^{2}+w_{2}^{2}}$, where $w_{1}$ and $w_{2}$ represent the half width at half maximum for peaks $E_{1}$ and $E_{2}$, respectively. We note that the EDC fitting in panel (g) yields the standard deviation of the peak positions much smaller (less than $1 \mathrm{meV}$ ) than the error bars shown in panel (h).

a vanishing or even negative area of peak $E_{2}$, and $\Delta=E_{1}-E_{2} \leq 3 \mathrm{meV}$, which is negligible compared to the width of the Lorentzian peaks. In short, the gaplessness of the Dirac cone at temperatures above $11 \mathrm{~K}$ is well established.

Assuming a linear relation between this exchange splitting and the magnetic moment, the gap should be well described by a power-law curve [48] $\Delta \sim E_{0}\left(1-T / T_{0}\right)^{2 \beta}$, where $E_{0}$ represents the saturated exchange splitting energy at $T=0 \mathrm{~K}$. Fitting the $\Delta(T)$ curve with this power-law function yields $T_{0}=11 \pm 1 \mathrm{~K}$ and $\beta=0.23 \pm 0.02$. The fitted onset temperature $T_{0}$ matches the susceptibilityderived Curie temperature well within the fitting error. The saturated exchange splitting energy is fitted as $E_{0}=33 \pm 1 \mathrm{meV}$. We thus established a FM-induced Dirac-point gap in the $\mathrm{S}$ termination of $\mathrm{MnBi}_{8} \mathrm{Te}_{13}$. It is noteworthy that, although Dirac-point gaps have been reported for other members of the $\mathrm{Mn}-\mathrm{Bi}-\mathrm{Te}$ family [25,35-37], these observations are still controversial
[24,28,30,32-34]. In particular, all the reported gaps remain open above the magnetic transition temperature, contradicting the scenario of the restoration of time-reversal symmetry. Consequently, our results - that a TSS Dirac cone gap decreases monotonically with increasing temperature and closes right at $T_{C}$, forming a gapless Dirac cone-represent the first smoking-gun evidence of TSSs gapped by the magnetic order among all known magnetic topological materials.

One may wonder why this magnetic gap can be observed in $\mathrm{FM} \mathrm{MnBi}_{8} \mathrm{Te}_{13}$ but not in its other AFM sisters. While the mechanism of the gapless Dirac cone in AFM Mn-Bi-Te family members remains an open issue, in the study of the surface band structure of $\mathrm{MnBi}_{6} \mathrm{Te}_{10}$, we pointed out that surface-bulk band hybridization may cause the surface Dirac cone distribution to extend to the bulk [28]. In an AFM background, this extension could result in much-reduced effective magnetic moments, which the surface state can feel, thus a (nearly) gapless Dirac cone 
with AFM order. In a FM background, the redistribution of the surface state does not change the effective magnetic moments, leading to the gapped Dirac cone.

\section{SURFACE ANOMALOUS HALL CONDUCTANCE AS A SIGNATURE OF AXION INSULATOR}

So far, we have demonstrated a magnetic gap at the $S$ termination and a gapless feature at the QQQ termination of $\mathrm{MnBi}_{8} \mathrm{Te}_{13}$, rendering the material a "half-magnetic topological insulator." To further identify its topological nature, we next theoretically analyze the surface anomalous Hall conductance (AHC) of this gapped surface and the corresponding experimental signatures. Because of the inversion symmetry, the band structure of $\mathrm{MnBi}_{8} \mathrm{Te}_{13}$ may be characterized by a higher-order topological invariant, i.e., the $Z_{4}$ number (the symmetry indicator of inversion [49,50]). Our explicit computation shows that $\mathrm{MnBi}_{8} \mathrm{Te}_{13}$ has $Z_{4}=2$, in agreement with a previous study [40] (Fig. S10 and Table S1 in Ref. [39]). For a FM compound, while $Z_{4}=1$ or 3 implies a Weyl semimetal, $Z_{4}=2$ corresponds to an axion insulator or a 3D Chern insulator, with distinct surface AHC behavior [13]. Therefore, we compute the surface $\mathrm{AHC}$ by integrating the local Chern numbers through surface-related layers for two-dimensional slabs of $\mathrm{MnBi}_{8} \mathrm{Te}_{13}$, expressed as

$\sigma_{x y}(L)=\frac{e^{2}-4 \pi}{h} \frac{\pi}{A} \operatorname{Im} \sum_{l=0}^{L} \frac{1}{N_{k}} \sum_{k} \sum_{v v^{\prime} c} X_{v c k} Y_{v^{\prime} c k}^{\dagger} \rho_{v v^{\prime} k}(l)$,

where $X$ and $Y$ are the position operators along the $x$ and $y$ directions, respectively, which are directly computed from the velocity operators through $X(Y)_{v c k}=\left\langle\psi_{v k}\left|i \hbar v_{x(y)}\right| \psi_{c k}\right\rangle /\left(E_{c k}-E_{v k}\right)$. The indices $v$ and $c$ denote the valence and conduction bands, respectively. Here, $\rho_{v v^{\prime}}(l)$ is the projection matrix on the corresponding layer $l$, which implies a summation over all atoms within a vdW layer. To uncover the locality of the surface AHC, we construct two slabs with different thicknesses. For the $\mathrm{S}$ termination of a 16-vdW-layer slab, when $E_{F}$ lies in the gap of this surface, the layer-integrated AHC reaches about $e^{2} / 2 h$ at the second layer from the $\mathrm{S}$ termination [Fig. 4(a)]. On the other hand, the metallic QQQ surface does not have a well-defined surface Chern number because $E_{F}$ inevitably cuts the surface bands (Fig. S11). In comparison, the slab with $17 \mathrm{vdW}$ layers is symmetric and has a global band gap, leading to a well-defined integer-quantized Chern number for the whole slab, i.e., $C=1$. Figure 4(b) clearly shows that both top and bottom surfaces contribute a half-quantized AHC, while the internal layers do not contribute to the global AHC. In the bulk, there is an oscillation around $e^{2} / 2 h$ with a period of the unit cell thickness (four vdW layers) starting from the fourth layer from the surface. Therefore, the half-quantized AHC of $\mathrm{MnBi}_{8} \mathrm{Te}_{13}$ is a local quantity at the $\mathrm{S}$ termination, indicating an axion-insulator phase.

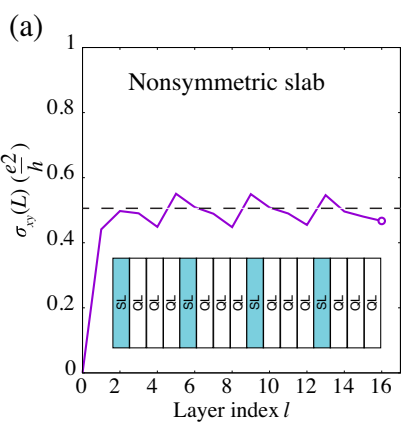

(c)
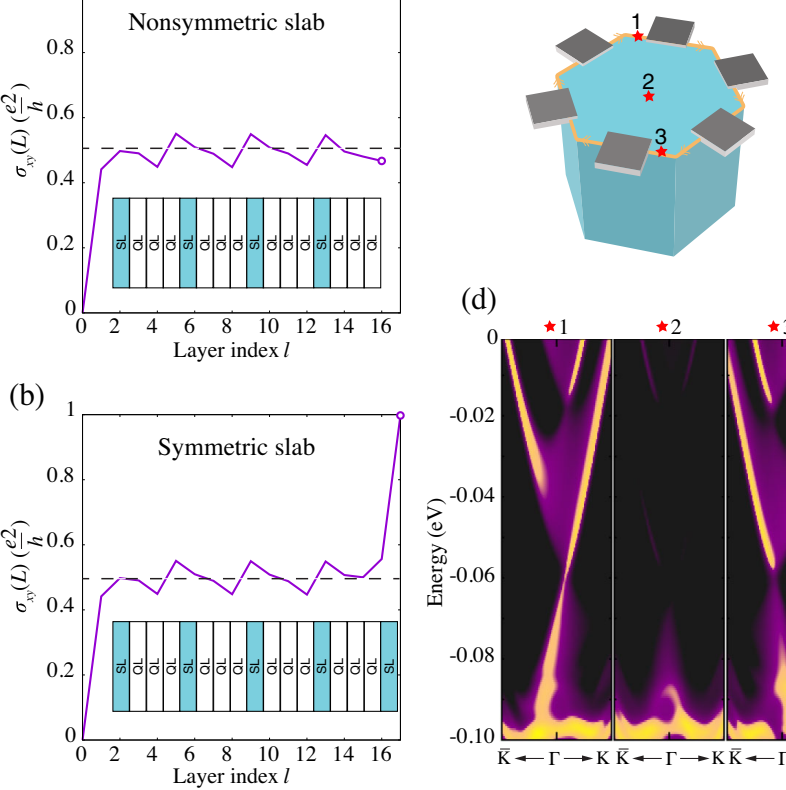

(d)

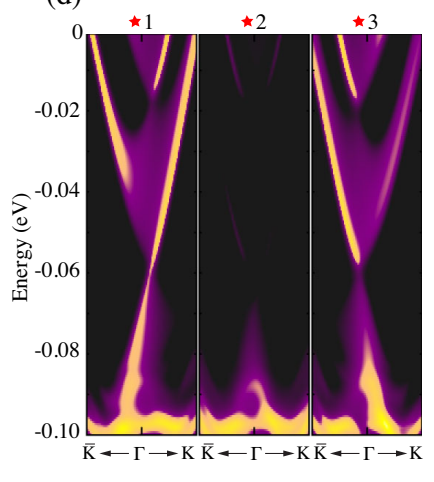

FIG. 4. Half-quantized surface $\mathrm{AHC}$ at the $\mathrm{S}$ termination. (a,b) Integrated, layer-projected, anomalous Hall conductance for the slabs with 16 and $17 \mathrm{vdW}$ layers, respectively. The 16-layer slab is not symmetric, containing four unit cells as a half-magnetic topological insulator, while the 17-layer slab is symmetric with a global band gap and nontrivial Chern number, as shown in the insets. (c) Schematic plot for the nonlocal transport measurement with a hexagonal six-contact-probing setup. (d) Spectral functions at three spots denoted in panel (c), showing chiral hinge states at the $\mathrm{S}$ termination that manifest the half-QAH effect.

Though direct experimental measurement of the half-quantized surface AHC is challenging for various reasons-including sample quality, possible electrode scattering, actual size of the surface gap, and the electron chemical potential in the sample-numerical validation in $\mathrm{MnBi}_{2} \mathrm{Te}_{4} /\left(\mathrm{Bi}_{2} \mathrm{Te}_{3}\right)_{n}$ [13] and an experimental proposal based on nonlocal transport measurement have recently been provided [14], with a hexagonal six-contact-probing setup shown in Fig. 4(c). In the case of $\mathrm{MnBi}_{8} \mathrm{Te}_{13}$, as discussed above, magnetism opens a gap in the $\mathrm{S}$ termination surface. We compute the spectral functions at the hinges and the center of the top surface ( $\mathrm{S}$ termination) [Fig. 4(d)], validating the existence of the chiral hinge state at the $\mathrm{S}$ termination. It clearly shows that, although the center of the $\mathrm{S}$ termination [point 2 in Fig. 4(c)] has a gap, nonvanishing chiral states exist near the boundary formed by the $x-y$ top surface and the $x-z$ side surface, i.e., points 1 and 3 , with opposite velocity. Such chiral hinge states are embedded within the side surface states, causing imbalanced density of states at the two sides of the Dirac cone. In this sense, $\mathrm{MnBi}_{8} \mathrm{Te}_{13}$ is an excellent candidate for observing the signature of the long-sought axion insulator and topological magnetoelectric effect. 


\section{ACKNOWLEDGMENTS}

We thank $\mathrm{Ni} \mathrm{Ni}$, Haizhou $\mathrm{Lu}$, and $\mathrm{Hu} \mathrm{Xu}$ for helpful discussions. This work is supported by National Key R\&D Program of China (Grants No. 2020YFA0308900 and No. 2019YFA0704900), the National Natural Science Foundation of China (NSFC) (Grants No. 12074163, No. 12074161, No. 11804144, No. 11674149, No. 11874195, and No. 11804402), the Shenzhen High-level Special Fund (Grants No. G02206304 and No. G02206404), the Guangdong Innovative and Entrepreneurial Research Team Program (Grants No. 2019ZT08C044, No. 2017ZT07C062, and No. 2016ZT06D348), Shenzhen Science and Technology Program (Grant No. KQTD201 90929173815000), the University Innovative Team in Guangdong Province (No. 2020KCXTD001), the Technology and Innovation Commission of Shenzhen Municipality (No. JCYJ20150630145302240 and No. KYTDPT20181011104202253), the Highlight Project (No. PHYS-HL-2020-1) of the College of Science at SUSTech, Guangdong Provincial Key Laboratory for Computational Science and Material Design under Grant No. 2019B030301001, and Center for Computational Science and Engineering of Southern University of Science and Technology. ARPES measurements were performed with the approval of the Proposal Assessing Committee of the Hiroshima Synchrotron Radiation Center (Proposals No. 19BG044, No. 19BU002, No. 19BU005, and No. 19BU012).

\section{APPENDIX: MATERIALS AND METHODS}

\section{Sample growth}

$\mathrm{MnBi}_{8} \mathrm{Te}_{13}$ single crystals were grown by the conventional high-temperature solution method using $\mathrm{Bi}_{2} \mathrm{Te}_{3}$ as the flux. Mn (purity 99.98\%), Bi (purity 99.999\%), and Te $(99.999 \%)$ blocks were placed in an alumina crucible with a molar ratio of $\mathrm{Mn}: \mathrm{Bi}: \mathrm{Te}=1: 12.3: 19.4$. Then, the alumina crucible was sealed in a quartz tube under the argon environment. The assembly was first heated up in a box furnace to $950^{\circ} \mathrm{C}$, held for $10 \mathrm{hrs}$, and then cooled down slowly to $574^{\circ} \mathrm{C}$ over $120 \mathrm{hrs}$. After this heating procedure, the quartz tube was taken out quickly and then decanted into the centrifuge to remove the excess flux from the single crystals. Because the temperature window for the growth of $\mathrm{MnBi}_{8} \mathrm{Te}_{13}$ is very narrow and $\mathrm{Bi}_{2} \mathrm{Te}_{3}$ is an inevitable by-product, we cut the single crystals into thin pieces and checked using single-crystal $\mathrm{x}$-ray diffraction on both sides to select only the pure phase of $\mathrm{MnBi}_{8} \mathrm{Te}_{13}$ single crystals.

\section{Transport and magnetic measurements}

The structure of the crystals was characterized by x-ray diffraction with $\mathrm{Cu} K a$ radiation at room temperature using a Rigaku Miniex diffractometer. The diffraction pattern can be well indexed by the $(00 l)$ reflections with
$\Delta_{2 \theta} \sim 2^{\circ}$ for adjacent peaks, especially at lower angles $\left(<20^{\circ}\right)$. Resistivity measurements were performed by a Quantum Design (QD) Physical Properties Measurement System (PPMS) with a standard six-probe method, using a drive current of $8 \mathrm{~mA}$. The current flows in the $a b$ plane, and the magnetic field is perpendicular to the current direction. Magnetic measurements were performed using the QD Magnetic Property Measurement System (MPMS) with the Vibrating Sample Magnetometer (VSM) mode. Temperature-dependent magnetization results were collected with an external magnetic field of $100 \mathrm{Oe}$, both along and perpendicular to the (001) direction of the sample.

\section{ARPES measurement}

The $\mu$-laser-ARPES [44] measurements were performed at the Hiroshima Synchrotron Radiation Center (HSRC), Hiroshima, Japan with a VG Scienta R4000 electron analyzer and a photon energy of $6.36 \mathrm{eV}$. The energy and angular resolution were better than $3 \mathrm{meV}$ and less than $0.05^{\circ}$, respectively. Samples were cleaved in situ along the (001) crystal plane under ultrahigh-vacuum conditions with pressure better than $5 \times 10^{-11}$ mbar and temperatures below $20 \mathrm{~K}$.

\section{First-principles calculations}

DFT calculations [51,52] were conducted by using the projector-augmented wave (PAW) pseudopotentials [53], and exchange correlation was described by the PerdewBurke-Ernzerhof (PBE) version of the GGA functional $[54,55]$ as implemented in the Vienna ab-initio Simulation Package (VASP) [56]. Considering the transition-metal element $\mathrm{Mn}$ in $\mathrm{MnBi}_{8} \mathrm{Te}_{13}$, the $\mathrm{PBE}+\mathrm{U}$ functional with $U=5 \mathrm{eV}$ was used for Mn $3 d$ orbitals for all the results in this work [57]. The $k$-mesh, energy cutoff, and total energy tolerance for the self-consistent calculations were $5 \times 5 \times 5,500 \mathrm{eV}$, and $10^{-5} \mathrm{eV}$, respectively. The experimental lattice constants $\left(a_{0}=4.37 \AA\right.$ and $\left.c_{0}=132.32 \AA\right)$ were taken, while the atomic positions were fully relaxed until the force on each atom was less than $10^{-2} \mathrm{eV} / \AA$. Spin-orbit coupling was included in the calculations selfconsistently. We constructed Wannier representations by projecting the Bloch states from the first-principles calculations of bulk materials onto $\mathrm{Mn}-d, \mathrm{Bi}-p$, and Te-s orbitals. The TSS, as well as the surface anomalous Hall conductivity, were calculated in tight-binding models constructed by these Wannier representations, as implemented in the WannierTools package [58-61].

[1] M. M. Otrokov et al., Prediction and Observation of an Antiferromagnetic Topological Insulator, Nature (London) 576, 416 (2019). 
[2] E. D. L. Rienks et al., Large Magnetic Gap at the Dirac Point in $\mathrm{Bi}_{2} \mathrm{Te}_{3} / \mathrm{MnBi}_{2} \mathrm{Te}_{4}$ Heterostructures, Nature (London) 576, 423 (2019).

[3] Y. Deng et al., Quantum Anomalous Hall Effect in Intrinsic Magnetic Topological Insulator $\mathrm{MnBi}_{2} \mathrm{Te}_{4}$, Science 367, 895 (2020).

[4] C. Liu et al., Robust Axion Insulator and Chern Insulator Phases in a Two-Dimensional Antiferromagnetic Topological Insulator, Nat. Mater 19, 522 (2020).

[5] R. S. K. Mong, A. M. Essin, and J. E. Moore, Antiferromagnetic Topological Insulators, Phys. Rev. B 81, 245209 (2010).

[6] E. Liu et al., Giant anomalous Hall Effect in a Ferromagnetic Kagome-Lattice Semimetal, Nat. Phys. 14, 1125 (2018).

[7] D. F. Liu et al., Magnetic Weyl Semimetal Phase in a Kagomé Crystal, Science 365, 1282 (2019).

[8] N. Morali, R. Batabyal, P. K. Nag, E. Liu, Q. Xu, Y. Sun, B. Yan, C. Felser, N. Avraham, and H. Beidenkopf, FermiArc Diversity on Surface Terminations of the Magnetic Weyl Semimetal $\mathrm{CO}_{3} \mathrm{Sn}_{2} \mathrm{~S}_{2}$, Science 365, 1286 (2019).

[9] Y. Tokura, K. Yasuda, and A. Tsukazaki, Magnetic Topological Insulators, Nat. Rev. Phys. 1, 126 (2019).

[10] N. P. Armitage and L. Wu, On the Matter of Topological Insulators as Magnetoelectrics, SciPost Phys. 6, 046 (2019).

[11] C.-Z. Chang et al., Experimental Observation of the Quantum Anomalous Hall Effect in a Magnetic Topological Insulator, Science 340, 167 (2013).

[12] C. Z. Chang, W. Zhao, D. Y. Kim, H. Zhang, B. A. Assaf, D. Heiman, S.-C. Zhang, C. Liu, M. H. W. Chan, and J. S. Moodera, High-Precision Realization of Robust Quantum Anomalous Hall State in a Hard Ferromagnetic Topological Insulator, Nat. Mater. 14, 473 (2015).

[13] M. Gu, J. Li, H. Sun, Y. Zhao, C. Liu, J. Liu, and Q. Liu, Spectral Signatures of the Surface Anomalous Hall Effect in Magnetic Axion Insulators, arXiv:2005.13943.

[14] R. Chen, S. Li, H.-P. Sun, Y. Zhao, H.-Z. Lu, and X. C. Xie, Using Nonlocal Surface Transport to Identify the Axion Insulator, arXiv:2005.14074.

[15] X.-L. Qi, T. L. Hughes, and S.-C. Zhang, Topological Field Theory of Time-Reversal Invariant Insulators, Phys. Rev. B 78, 195424 (2008).

[16] A. M. Essin, J. E. Moore, and D. Vanderbilt, Magnetoelectric Polarizability and Axion Electrodynamics in Crystalline Insulators, Phys. Rev. Lett. 102, 146805 (2009).

[17] J. Ge et al., High-Chern-Number and High-Temperature Quantum Hall Effect without Landau Levels, Natl. Sci. Rev. 7, 1280 (2020).

[18] Y. Gong et al., Experimental Realization of an Intrinsic Magnetic Topological Insulator, Chin. Phys. Lett. 36, 076801 (2019).

[19] J. Li, Y. Li, S. Du, Z. Wang, B.-L. Gu, S.-C. Zhang, K. He, W. Duan, and Y. Xu, Intrinsic Magnetic Topological Insulators in van der Waals Layered MnBi2Te4-Family Materials, Sci. Adv. 5, eaaw5685 (2019).

[20] M. M. Otrokov, I. P. Rusinov, M. Blanco-Rey, M. Hoffmann, A. Yu. Vyazovskaya, S. V. Eremeev, A. Ernst, P. M. Echenique, A. Arnau, and E. V. Chulkov, Unique Thickness-Dependent Properties of the van der Waals
Interlayer Antiferromagnet $\mathrm{MnBi}_{2} \mathrm{Te}_{4}$ Films, Phys. Rev. Lett. 122, 107202 (2019).

[21] H. Sun, B. Xia, Z. Chen, Y. Zhang, P. Liu, Q. Yao, H. Tang, Y. Zhao, H. Xu, and Q. Liu, Rational Design Principles of the Quantum Anomalous Hall Effect in Superlatticelike Magnetic Topological Insulators, Phys. Rev. Lett. 123, 096401 (2019).

[22] D. Zhang, M. Shi, T. Zhu, D. Xing, H. Zhang, and J. Wang, Topological Axion States in the Magnetic Insulator $\mathrm{MnBi}_{2} \mathrm{Te}_{4}$ with the Quantized Magnetoelectric Effect, Phys. Rev. Lett. 122, 206401 (2019).

[23] K. N. Gordon, H. Sun, C. Hu, A. G. Linn, H. Li, Y. Liu, P. Liu, S. Mackey, Q. Liu, N. Ni, and D. Dessau, Strongly Gapped Topological Surface States on Protected Surfaces of Antiferromagnetic $\mathrm{MnBi}_{4} \mathrm{Te}_{7}$ and $\mathrm{MnBi}_{6} \mathrm{Te}_{10}$, arXiv: 1910.13943.

[24] L.X. Xu, et al., Persistent Gapless Surface States in $\mathrm{MnBi}_{2} \mathrm{Te}_{4} / \mathrm{Bi}_{2} \mathrm{Te}_{3}$ Superlattice Antiferromagnetic Topological Insulator, arXiv:1910.11014.

[25] N. H. Jo, L.-L. Wang, R.-J. Slager, J. Yan, Y. Wu, K. Lee, B. Schrunk, A. Vishwanath, and A. Kaminski, Intrinsic Axion Insulating Behavior in Antiferromagnetic $\mathrm{MnBi}_{6} \mathrm{Te}_{10}$, Phys. Rev. B 102, 045130 (2020).

[26] S. Tian et al., Magnetic Topological Insulator $\mathrm{MnBi}_{6} \mathrm{Te}_{10}$ with a Zero-Field Ferromagnetic State and Gapped Dirac Surface States, Phys. Rev. B 102, 035144 (2020).

[27] R. C. Vidal et al., Topological Electronic Structure and Intrinsic Magnetization in $\mathrm{MnBi}_{4} \mathrm{Te}_{7}: A \mathrm{Bi}_{2} \mathrm{Te}_{3}$ Derivative with a Periodic Mn Sublattice, Phys. Rev. X 9, 041065 (2019).

[28] X.-M. Ma et al., Hybridization-Induced Gapped, and Gapless States on the Surfaces of Magnetic Topological Insulators, Phys. Rev. B 102, 245136 (2020).

[29] C. Hu et al., A van der Waals Antiferromagnetic Topological Insulator with Weak Interlayer Magnetic Coupling, Nat. Commun. 11, 97 (2020).

[30] Y. Hu et al., Universal Gapless Dirac Cone and Tunable Topological States in $\left(\mathrm{MnBi}_{2} \mathrm{Te}_{4}\right) \mathrm{m}\left(\mathrm{Bi}_{2} \mathrm{Te}_{3}\right) \mathrm{n}$ Heterostructures, Phys. Rev. B 101, 161113 (2020).

[31] X. Wu et al., Distinct Topological Surface States on the Two Terminations of $\mathrm{MnBi}_{4} \mathrm{Te}_{7}$, Phys. Rev. X 10, 031013 (2020).

[32] Y. J. Chen et al., Topological Electronic Structure and Its Temperature Evolution in Antiferromagnetic Topological Insulator $\mathrm{MnBi}_{2} \mathrm{Te}_{4}$, Phys. Rev. X 9, 041040 (2019).

[33] Y.-J. Hao et al., Gapless Surface Dirac Cone in Antiferromagnetic Topological Insulator $\mathrm{MnBi}_{2} \mathrm{Te}_{4}$, Phys. Rev. X 9 , 041038 (2019).

[34] P. Swatek, Y. Wu, L.-L. Wang, K. Lee, B. Schrunk, J. Yan, and A. Kaminski, Gapless Dirac Surface States in the Antiferromagnetic Topological Insulator $\mathrm{MnBi}_{2} \mathrm{Te}_{4}$, Phys. Rev. B 101, 161109 (2020).

[35] A. M. Shikin et al., Nature of the Dirac Gap Modulation and Surface Magnetic Interaction in Axion Antiferromagnetic Topological Insulator $\mathrm{MnBi}_{2} \mathrm{Te}_{4}$, Sci. Rep. 10, 13226 (2020).

[36] H. Li et al., Dirac Surface States in Intrinsic Magnetic Topological Insulators $\mathrm{EuSn}_{2} \mathrm{As}_{2}$ and $\mathrm{MnBi}_{2 n} \mathrm{Te}_{3 n+1}$, Phys. Rev. X 9, 041039 (2019). 
[37] X.-M. Ma et al., Spectroscopic Realization of Large Surface Gap in a Doped Magnetic Topological Insulator, arXiv:2004 .09123.

[38] I. I. Klimovskikh et al., Tunable 3D/2D Magnetism in the $\left(\mathrm{MnBi}_{2} \mathrm{Te}_{4}\right)\left(\mathrm{Bi}_{2} \mathrm{Te}_{3}\right) \mathrm{m}$ Topological Insulators Family, npj Quantum Mater. 5, 54 (2020).

[39] See Supplemental Material at http://link.aps.org/ supplemental/10.1103/PhysRevX.11.011039 for more information on $\mathrm{x}$-ray diffraction, magnetic and electric properties, comparison between DFT and ARPES results, and the determination of the band topology.

[40] C. Hu et al., Realization of an Intrinsic Ferromagnetic Topological State in $\mathrm{MnBi}_{8} \mathrm{Te}_{13}$, Sci. Adv. 6, eaba4275 (2020).

[41] J. Q. Yan, S. Okamoto, M. A. McGuire, A. F. May, R. J. McQueeney, and B.C. Sales, Evolution of Structural, Magnetic, and Transport Properties in $\mathrm{MnBi}_{2}-\mathrm{Sb}_{x} \mathrm{Te}_{4}$, Phys. Rev. B 100, 104409 (2019).

[42] L. Ding, C. Hu, F. Ye, E. Feng, N. Ni, and H. Cao, Crystal and Magnetic Structures of Magnetic Topological Insulators $\mathrm{MnBi}_{2} \mathrm{Te}_{4}$ and $\mathrm{MnBi}_{4} \mathrm{Te}_{7}$, Phys. Rev. B 101, 020412 (2020).

[43] J. Q. Yan, Y. H. Liu, D. S. Parker, Y. Wu, A. A. Aczel, M. Matsuda, M. A. McGuire, and B. C. Sales, A-Type Antiferromagnetic Order in $\mathrm{MnBi}_{4} \mathrm{Te}_{7}$ and $\mathrm{MnBi}_{6} \mathrm{Te}_{10}$ Single Crystals, Phys. Rev. Mater. 4, 054202 (2020).

[44] H. Iwasawa, E. F. Schwier, M. Arita, A. Ino, H. Namatame, M. Taniguchi, Y. Aiura, and K. Shimada Development of Laser-Based Scanning Micro-ARPES System with Ultimate Energy and Momentum Resolutions, Ultramicroscopy 182, 85 (2017).

[45] It is still controversial whether a topological surface gap exists in $\mathrm{MnBi}_{2} \mathrm{Te}_{4}$. While one recent work (Ref. [36]) reports two types of gaps that persist at AFM and PM phases (nonmagnetic), we always observe a gapless Dirac cone in $\mathrm{MnBi}_{2} \mathrm{Te}_{4}$ at AFM and PM phases (Ref. [24]).

[46] P. Zhang, P. Richard, T. Qian, Y.-M. Xu, X. Dai, and H. Ding,A Precise Method for Visualizing Dispersive Features in Image Plots, Rev. Sci. Instrum. 82, 043712 (2011).

[47] C. J. Powell, A. Jablonski, I. S. Tilinin, S. Tanuma, and D. R. Penn, Surface Sensitivity of Auger-Electron Spectroscopy and X-Ray Photoelectron Spectroscopy, J. Electron Spectrosc. Relat. Phenom. 98-99, 1 (1999).

[48] D. A. Estyunin et al., Signatures of Temperature Driven Antiferromagnetic Transition in the Electronic Structure of
Topological Insulator $\mathrm{MnBi}_{2} \mathrm{Te}_{4}$, APL Mater. 8, 021105 (2020).

[49] J. Kruthoff, J. de Boer, J. van Wezel, C. L. Kane, and R.-J. Slager, Topological Classification of Crystalline Insulators through Band Structure Combinatorics, Phys. Rev. X 7, 041069 (2017).

[50] W. Haruki, P. H. Chun, and V. Ashvin, Structure and Topology of Band Structures in the 1651 Magnetic Space Groups, Sci. Adv. 4, eaat8685 (2018).

[51] P. Hohenberg and W. Kohn, Inhomogeneous Electron Gas, Phys. Rev. 136, B864 (1964).

[52] W. Kohn and L. J. Sham, Self-Consistent Equations Including Exchange and Correlation Effects, Phys. Rev. 140, A1133 (1965).

[53] G. Kresse and D. Joubert, From Ultrasoft Pseudopotentials to the Projector Augmented-Wave Method, Phys. Rev. B 59, 1758 (1999).

[54] J. P. Perdew, K. Burke, and M. Ernzerhof, Generalized Gradient Approximation Made Simple, Phys. Rev. Lett. 77, 3865 (1996).

[55] J. P. Perdew, K. Burke, and M. Ernzerhof, Generalized Gradient Approximation Made Simple, Phys. Rev. Lett. 77, 3865 (1996); Phys. Rev. Lett.78, 1396 (1997).

[56] G. Kresse and J. Furthmüller, Efficient Iterative Schemes for ab initio Total-Energy Calculations Using a Plane-Wave Basis Set, Phys. Rev. B 54, 11169 (1996).

[57] S. L. Dudarev, G. A. Botton, S. Y. Savrasov, C. J. Humphreys, and A. P. Sutton, Electron-Energy-Loss Spectra and the Structural Stability of Nickel Oxide: An LSDA + U Study, Phys. Rev. B 57, 1505 (1998).

[58] N. Marzari and D. Vanderbilt, Maximally Localized Generalized Wannier Functions for Composite Energy Bands, Phys. Rev. B 56, 12847 (1997).

[59] I. Souza, N. Marzari, and D. Vanderbilt, Maximally Localized Wannier Functions for Entangled Energy Bands, Phys. Rev. B 65, 035109 (2001).

[60] A. A. Mostofi, J. R. Yates, G. Pizzi, Y.-S. Lee, I. Souza, D. Vanderbilt, and N. Marzari An Updated Version of Wannier90: A Tool for Obtaining Maximally-Localised Wannier Functions, Comput. Phys. Commun. 185, 2309 (2014).

[61] Q. Wu, S. Zhang, H.-F. Song, M. Troyer, and A. A. Soluyanov, WannierTools: An Open-Source Software Package for Novel Topological Materials, Comput. Phys. Commun. 224, 405 (2018). 